\title{
Glycated Hemoglobin and Lipid Profile Association Among Pregnant Women in Saudi Arabian Population
}

\author{
Farah Anjum $^{1 * \mathbb{D}}$, Rana Zaini $^{1,2}$, Ashjan Shami ${ }^{1}$, Amani Rehaili ${ }^{1}$, Rahma Kufia $^{3}$
}

\begin{abstract}
Objectives: Glycated hemoglobin (HbAlc) is the most commonly used glycemic index among diabetic patients. The present study sought to investigate the relationship between $\mathrm{HbAlc}$ and lipid parameters among healthy pregnancies and gestational diabetic cases. Materials and Methods: Seventy-five Saudi pregnant women within the age group of 19 to 43 years were selected for this crosssectional study. The participants were categorized into with and without gestational diabetes (GDM), all of whom were non-smokers; in addition, they neither had a previous history of chronic illnesses nor were they on any medications which could alter lipid profile and HbAlc concentrations. Totally, $75 \mathrm{~g}$ of oral glucose tolerance test (OGTT) was utilized to identify 25 women with GDM. All biochemical tests including HbAlc, fasting blood sugar (FBG), total cholesterol (TC), low-density lipoprotein (LDL-cholesterol), triglycerides (TG), and high-density lipoprotein (HDL-cholesterol) were performed by a biochemical auto-analyzer. The collected data were statistically analyzed using a paired student's $t$ test. The $P$ values of $<0.05$ were considered statistically significant on all the analyses related to healthy pregnancies and those associated with GDM.

Results: The HbA1c and FBG values demonstrated significant correlation with GDM $(P<0.001$ each). However, TC, LDL-cholesterol, and TG failed to indicate any significant difference between the 2 groups. Conversely, HDL-cholesterol and the ratio of TG and HDLcholesterol revealed remarkable differences between GDM and healthy participants $(P<0.05$ each $)$.

Conclusions: In general, the blood levels of glucose, HbA1c, TG, and HDL-cholesterol are of paramount significance in GDM. Predictably, HbA1c can be employed as one of the markers in evaluating the risk factors in developing dyslipidemia among pregnant women.

Keywords: Gestational diabetes mellitus, Lipid profile, Glycated hemoglobin
\end{abstract}

\section{Introduction}

Gestational diabetes (GDM) is a potentially serious condition which affects several pregnancies worldwide, the prevalence of which has growingly increased by 2 to 3 folds in the last few decades. GDM is defined as the impaired glucose tolerance which causes high levels of blood glucose and is essentially identified during pregnancy (1). It occurs due to the onset of the series related to the major metabolic disturbance in carbohydrate metabolism. In addition, abnormal levels of plasma lipids and lipoprotein concentrations are typically encountered in these individuals. It is a well-known fact that major alterations in carbohydrate, lipid, and amino acid metabolism take place even in normal pregnant women mainly due to the hormonal changes and mild insulin resistance (2). Those women who had limited beta cell capacity for compensating the pregnancy-induced insulin resistance due to a variation in the genes involved in insulin secretion and carbohydrate utilization are likely to develop GDM (3). Lipid metabolism in normal pregnancy undergoes adaptive changes. Physiologically, a 2 to 3 times increment in plasma triglycerides (TG) is found in second and third trimesters of gestation. This is frequently accompanied by a lesser degree of increase in the levels of the total cholesterol (TC), high-density lipoprotein (HDL-cholesterol), and low-density lipoprotein (LDLcholesterol). Further, postpartum, TC, HDL-cholesterol, and TG demonstrate a decline while the LDL-cholesterol level tends to remain unchanged (4-6). Previous studies detected a relationship between the parameters of the lipid, the degree of glucose tolerance, the function of the endothelial cell, and risk of developing cardiovascular diseases among pregnant females. Furthermore, it was revealed that serum TG values observed during the first trimester of gestation have a good predictive utility for determining glucose tolerance during the entire phase of pregnancy (5). An increased level of oxidative stress and inflammation during pregnancy may have a consequence on long-term cardiovascular health, especially in women having the predisposition to other risk factors responsible for a variety of cardiovascular illnesses (7).

Glycated hemoglobin (HbAlc) is a commonly used indicator of blood glucose levels in diabetics. It reflects the previous 8-10 weeks average level of plasma glucose (8). The HbA1c helps to speculate the risk for developing the array of diabetic complications, along with indicating 
the mean blood glucose level (8). A significant rise in HbA1c level is considered to be an independent risk factor for causing derangement in lipid parameters which may lead to coronary heart disease and stroke in people with or without diabetes (9). Therefore, a high value of $\mathrm{HbA1c}$ is linked to deranged lipid profile. Pregnancy is not an exception. In other words, diabetes and pregnancy have a cumulative role in causing a major disruption in metabolic rhythm which eventually results in developing an atherogenic lipid profile. This, in turn, has negative implications on both maternal and fetal health. Diabetic dyslipidemia is strongly related to the insulin resistance and abnormal lipid fractions leading to elevated levels of TG and LDL-cholesterol while decreasing the levels of HDLcholesterol. An approximate rise of $18 \%$ was estimated in the risk factors of several cardiovascular diseases for each $1 \%$ increment in absolute value of $\mathrm{HbAlc}$ among diabetic patients. Moreover, a positive association between HbAlc and the development of cardiovascular diseases was even observed in non-diabetic patients having a normal range of HbA1c values (10).

Alteration in the lipid profile is known to occur in GDM (11). Jiang et al indicated that increased levels of TC or TG during the prevalence of dyslipidemia among pregnant mothers are frequently associated with an outcome favoring a chance of having a preterm delivery (12). Premature birth or birth of an infant at less than 37 weeks of gestational age is the second most common cause of death in children less than 5 years of age. Additionally, it is related to the increased risk of developing derangement in metabolic processes later in life. These may further prove to be detrimental to both mother and child health (13).

So far, no conclusive studies have been conducted in Saudi Arabia regarding the association between the HbAlc and lipid profile among pregnant women. Therefore, this study focused on highlighting the relationship between the above-mentioned factors among pregnant women in Saudi Arabia and to prognosticate if HbAlc can be used as an independent marker of dyslipidemia among GDM cases.

\section{Materials and Methods}

The present cross-sectional study was implemented during (November) 2017-(February) 2018 and was approved by the Research Ethics Committee of the College of Applied Medical Sciences in Taif University and Primary Health Care Center in Mecca. In addition, all participants were informed about the research objectives and signed the consent form before completing the questionnaire and collecting the blood samples.

Based on the number of participants who completed the questionnaires and fulfilled the criteria for inclusion, a total of 75 pregnant women (50 healthy control vs. 25 gestational diabetic cases) within the age range of 19-43 years were recruited for the current study. Further, these participants were selected during their routine antenatal visit to a primary health care center in Mecca, Saudi Arabia and were within the end of the first trimester and beginning of the second trimester. Furthermore, they responded to the questionnaire containing information about their age, educational level, number of pregnancies, self-history and family history of GDM, as well as the family history of diabetes mellitus (DM), diet, lifestyle, and indulgence in physical exercises. Based on an initial screening for fasting blood glucose value and considering the risk factors, the GDM of 25 women was diagnosed based on 75 -g oral glucose tolerance test (OGTT) with 3 glucose values of venous blood using the hexokinase method. Moreover, the standard method of American Diabetes Association (ADA) was applied regarding having at least 2 values exceeding the set values $(95,180$, and $155 \mathrm{mg} / \mathrm{dL})$ in the venous sample (14). The selected patients had no previous history of medical illnesses such as hemoglobinopathy, iron-deficiency anemia, thyroid diseases, beforeconception diabetes mellitus, hypertension, renal, cardiac diseases. Additionally, they were all non-smokers while they were not on any medications or supplements that could modify lipid profile, insulin levels, and HbA1c concentrations.

Biochemical tests including $\mathrm{HbA} 1 \mathrm{c}$, fasting blood glucose (FBG), TC, LDL-cholesterol, TG, and HDL-cholesterol were conducted employing a biochemical automated analyzer in the primary health care centre in Mecca, Saudi Arabia. Samples for serum lipid profile parameters (i.e., TC, LDL-cholesterol, TG, and HDL-cholesterol) and FBG were collected after 10-12 hours of overnight fasting. In addition, blood withdrawn in plain (red-top) tube was first allowed to clot and then centrifuged at $3000 \mathrm{rpm}$ for 10 minutes in order to separate the serum. TC was enzymatically determined in a series of coupled reactions by the cholesterol oxidase/peroxidase aminophenazone (CHOD-PAP) colorimetric method and finally produced a color whose intensity was proportional to the cholesterol concentration at $500 \mathrm{~nm}$; TG was determined in a similar way by glycerophosphate oxidase and peroxidase (GPOPAP method) using the sequential enzymatic reactions which hydrolyzed TG into glycerol. Further reactions produced a color product which was measured at 500 nm. Furthermore, HDL-cholesterol was estimated by the action of polyethylene glycol (PEG)- coupled cholesteryl esterase-cholesterol oxidase ( $\mathrm{CHE}-\mathrm{CHO})$ and then, the absorption was measured at $600 \mathrm{~nm}$ after final peroxidase reaction. Moreover, LDL-cholesterol was determined to employ the enzymatic photometric method (CHE-CHO; peroxidase) and its concentration was photometrically calculated. Additionally, FBG was obtained by glucose oxidase (GOD) and peroxidase (POD) enzymatic method to form a red dye which was quantitated at $505 \mathrm{~nm}$. The reference range for serum lipids were considered as follows: TC $<200 \mathrm{mg} / \mathrm{dL} ;$ LDL-cholesterol $<130 \mathrm{mg} / \mathrm{dL} ; \mathrm{TG}<150$ $\mathrm{mg} / \mathrm{dL}$ and HDL-cholesterol $>50 \mathrm{mg} / \mathrm{dL}$; in addition, the reference range for FBG was considered to be $70-100 \mathrm{mg} /$ 
dL. Further, blood sample for HbA1c was collected in a lavender top tube with ethylenediaminetetraacetic acid (EDTA) and analyzed on the same day of collection. The reference range for $\mathrm{HbAlc}$ was considered from $4.3 \%$ to $6.0 \%$. Furthermore, the ratio of TG to HDL-cholesterol was calculated from their measured values; its reference range considered to be $<2$.

Finally, the collected data were statistically analyzed by the SPSS software, version 22 using the student's $t$ test. The $P$ values of $<0.05$ were considered statistically significant on all the measurements of healthy pregnant women and those with GDM.

\section{Results}

The mean age of 75 pregnant females included in this study was 30.6 years. Moreover, the mean age of 50 healthy control group was equal to 29.7 years and that of 25 women with GDM was estimated to be 31.4 years. The statistical analysis of demographics, obstetrics, health history, and lifestyle of those women with and without GDM are presented in Table 1. As shown, there is no significant variation $(P>0.05)$ in women with and without
GDM based on their age, educational level, the number of pregnancies, previous history of GDM, family history of GDM and DM, diet, lifestyle, and physical exercises.

Table 2 demonstrates the comparison of the lipid profile of women in both groups. Several variables are examined including TC, LDL-cholesterol, TG, HDL-cholesterol, as well as the ratio of TG and HDL-cholesterol. Based on their $P$ values, TC, LDL-cholesterol, and TG represent no remarkable variation between the 2 groups of pregnant women whereas HDL-cholesterol and the ratio of TG and HDL-cholesterol indicate a significant difference between the women with and without GDM $(P<0.05)$. Additionally, the means and standard deviations of

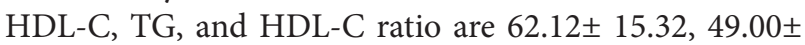
8.54 , as well as $1.76 \pm 0.90$ and $2.70 \pm 1.6$ in women with and without GDM, respectively.

In addition, the comparison of the mean values of FBG and HbAlc demonstrated in Table 3. As shown, there is a high degree of variation in these values between healthy pregnant women and women with GDM $(P<0.05)$. Further, the mean and standard deviation of FBG is equal to $93.92 \pm 6.14$ and $118.84 \pm 6.88$ and that of the HbAlc is

Table 1. The Comparison of Demographics, Obstetrics, Health History, and Lifestyle of Women Without GDM (control) and With GDM

\begin{tabular}{|c|c|c|c|c|c|c|}
\hline \multirow{2}{*}{ Variable } & & \multicolumn{2}{|c|}{ Non-GDM $(n=50)$} & \multicolumn{2}{|c|}{ GDM (n = 25) } & \multirow[t]{2}{*}{$P$ Value } \\
\hline & & Mean & SD & Mean & SD & \\
\hline \multirow[t]{2}{*}{ Age (y) } & & 29.7 & \pm 6.12 & 31.4 & \pm 6.06 & 0.15 \\
\hline & & $\mathbf{N}$ & $\%$ & $\mathbf{N}$ & $\%$ & \\
\hline \multirow{4}{*}{ Educational Level } & Illiterate & 3 & 6 & 3 & 12 & \\
\hline & Primary & 6 & 12 & 6 & 24 & 0.26 \\
\hline & Secondary & 24 & 48 & 6 & 24 & \\
\hline & Graduate & 17 & 34 & 10 & 40 & \\
\hline \multirow{9}{*}{ Gravida } & 1 & 10 & 20 & 0 & 0 & \\
\hline & 2 & 11 & 22 & 7 & 28 & \\
\hline & 3 & 8 & 16 & 3 & 12 & \\
\hline & 4 & 7 & 14 & 3 & 12 & \\
\hline & 5 & 7 & 14 & 3 & 12 & 0.09 \\
\hline & 6 & 3 & 6 & 6 & 24 & \\
\hline & 7 & 2 & 4 & 3 & 12 & \\
\hline & 8 & 1 & 2 & 0 & 0 & \\
\hline & 9 & 1 & 2 & 0 & 0 & \\
\hline \multirow{3}{*}{ Previous history of GDM } & Yes & 2 & 4 & 0 & 0 & \\
\hline & No & 45 & 90 & 19 & 76 & 0.61 \\
\hline & Do not know & 3 & 6 & 6 & 24 & \\
\hline \multirow{3}{*}{ Family history of GDM } & Yes & 17 & 34 & 13 & 52 & \\
\hline & No & 20 & 40 & 0 & 0 & 0.15 \\
\hline & Do not know & 13 & 26 & 12 & 48 & \\
\hline \multirow{2}{*}{ Family history of DM } & Yes & 31 & 62 & 18 & 72 & \\
\hline & No & 19 & 38 & 7 & 28 & 0.26 \\
\hline \multirow{3}{*}{ Food habit } & High calories & 5 & 10 & 7 & 28 & \\
\hline & Moderate calories & 32 & 64 & 15 & 60 & 0.39 \\
\hline & Low calories & 13 & 26 & 3 & 12 & \\
\hline \multirow{3}{*}{ Lifestyle } & Least active & 7 & 14 & 0 & 0 & \\
\hline & Moderately active & 23 & 46 & 9 & 36 & 0.28 \\
\hline & Highly active & 20 & 40 & 16 & 64 & \\
\hline \multirow{2}{*}{ Physical exercise } & Yes & 19 & 38 & 6 & 24 & \\
\hline & No & 31 & 62 & 19 & 76 & 0.29 \\
\hline
\end{tabular}

Note. SD: Standard deviation; GDM: Gestational diabetes. 
Table 2. The Comparison of Lipid Profile of Women Without GDM (control) and With GDM

\begin{tabular}{lccccc}
\hline \multirow{2}{*}{ Variable } & \multicolumn{2}{c}{ Non-GDM $(\mathbf{n = 5 0})$} & \multicolumn{2}{c}{ GDM $(\mathbf{n = 2 5})$} & \multirow{2}{*}{$\boldsymbol{P}$ value } \\
\cline { 2 - 5 } & Mean & SD & Mean & SD & \\
\hline TC, $\mathrm{mg} / \mathrm{dL}$ & 197.40 & \pm 40.53 & 185.12 & \pm 22.78 & 0.10 \\
$\mathrm{LDL}-\mathrm{C}, \mathrm{mg} / \mathrm{dL}$ & 114.22 & \pm 35.64 & 111.73 & \pm 17.26 & 0.68 \\
TG, $\mathrm{mg} / \mathrm{dL}$ & 105.26 & \pm 41.70 & 122.52 & \pm 51.50 & 0.15 \\
HDL-C, $\mathrm{mg} / \mathrm{dL}$ & 62.12 & \pm 15.32 & 49.00 & \pm 8.54 & $<0.001$ \\
TG: $\mathrm{HDL}-\mathrm{C}$ & 1.76 & \pm 0.90 & 2.70 & \pm 1.6 & 0.02 \\
\hline
\end{tabular}

Note. TC: total cholesterol; LDL: low-density lipoprotein; TG: triglyceride; HDL: high-density lipoprotein; GDM: gestational diabetes; SD; Standard deviation.

Table 3. The Comparison of Fasting Blood Glucose and Glycated Hemoglobin of Women Without GDM (control) and With GDM

\begin{tabular}{lccccc}
\hline \multirow{2}{*}{ Variable } & \multicolumn{2}{c}{ Non-GDM $(\mathbf{n = 5 0})$} & \multicolumn{2}{c}{ GDM $(\mathbf{n = 2 5})$} & \multirow{2}{*}{$\boldsymbol{P}$ value } \\
\cline { 2 - 5 } & Mean & SD & Mean & SD & \\
\hline FBG, mg/dL & 93.92 & \pm 40.53 & 118.84 & \pm 22.78 & $<0.001$ \\
HbAlc (\%) & 5.15 & \pm 0.90 & 6.33 & \pm 1.6 & $<0.001$ \\
\hline
\end{tabular}

Note. FBG: Fasting blood glucose; HbAlc: Glycated hemoglobin; GDM: gestational diabetes; SD; Standard deviation.

$5.15 \pm 0.33$ ) and $6.33 \pm 0.36$ in women with and without GDM, respectively.

\section{Discussion}

GDM has many deleterious immediate and long-term consequences for both mother and neonates. Pregnant women with high levels of blood glucose are susceptible to the risk of developing pre-eclampsia, preterm deliveries, cesarean delivery, as well as type-II diabetes later in their life. The neonates of these mothers are typically macrosomic due to the deranged intrauterine metabolic environment; that is, they develop respiratory distress and hyperbilirubinemia (15). Therefore, the ill-effects of GDM on lipid metabolism still remains ambiguous since GDM leads to alterations in maternal metabolic fuels, which is quite often associated with a variety of complications. Furthermore, the abnormalities of carbohydrate metabolism in GDM may affect lipid metabolism at large and both acting synergistically may act as important predictors of hypertension, dyslipidemia, and cardiovascular diseases (16). Accordingly, studies indicating an association between GDM and lipid profile are helpful in avoiding cardiovascular complications and decreasing the morbidity and mortality ratios of gestational diabetic mothers and neonates.

Several studies demonstrated marked alterations in values of plasma lipid profile and concentrations of lipoprotein (17-19). The current study was designed to investigate the lipid profile and glucose tolerance in GDM women and compare it with normal pregnant women. The results of previous studies revealed that plasma glucose values decrease during pregnancy due to its utilization by the growing fetus (20). In the present study, the fasting values of blood glucose in women with GDM were significantly increased $(P<0.001)$ and the actual levels were $118.84 \pm 6.88 \mathrm{mg} / \mathrm{dL}$ when compared with non-GDM levels (93.92 $\pm 6.14 \mathrm{mg} / \mathrm{dL})$. Taricco et al (21) obtained similar results respecting the increased levels of plasma glucose in maternal diabetes. Furthermore, Khan et al (22) found that the value of FBG was $112 \pm$ $8.70 \mathrm{mg} / \mathrm{dL}$ in GDM groups compared to $87 \pm 6.90 \mathrm{mg} /$ $\mathrm{dL}$ in healthy pregnant women $(P<0.001)$. Contrarily, Lenin et al (19) indicated no significant difference in FBG value among non-GDM and GDM groups among an Indian population. They reported an FBG measure of $72.45 \pm 7.23 \mathrm{mg} / \mathrm{dL}$ and $74.3 \pm 9.18 \mathrm{mg} / \mathrm{dL}(P=0.23)$ in the 2 groups, respectively. The results of the current study further revealed that glycated hemoglobin values elevated in GDM compared to the healthy pregnant women; in the present study, a mean of $6.33 \pm 0.36 \%$ and $5.15 \pm 0.33 \%$ were obtained in GDM and healthy controls, respectively. Ghafoor et al (23) compared HbAlc levels in non-diabetic pregnant women and GDM women of Pakistan and observed values of $6.04 \pm 0.16 \%$ and $7.50 \pm 0.43 \%$ among the 2 groups, respectively $(P<0.001)$. Moreover, Khan et al reported quite similar results in their study where they compared glycated hemoglobin of the 2 groups and found a value of $6.6 \pm 1.30 \%$ and $4.9 \pm 0.44 \%$ for GDM cases and healthy control women, respectively. Additionally, McGrowder et al (24) studied lipid profile and clinical characteristics of the women with GDM mellitus and preeclampsia in West Indies and represented that $\mathrm{HbAlc}$ was higher in women with GDM $(5.90 \pm 0.34 \%)$ compared to its value among the control group $(4.60 \pm 0.67 \%)$ with a $P$ value equal to 0.001 .

$\mathrm{HbAlc}$ is an indispensable means for managing longterm glycemic index and an invaluable marker of plasma lipid profile. Therefore, close observation of glycemic index using glycated hemoglobin is believed to be of high prognostic value, especially in diabetic patients who are predisposed to cardiovascular complications (25). It is well-known that an increased level of HbAlc poses an enormous amount of risk for the development of cardiovascular diseases including stroke in people who are already diagnosed as diabetic (26). The results of a research performed on 11092 non-diabetic patients as part of community-based population study revealed that high $\mathrm{HbAlc}$ numbers had a strong association with developing the highly favorable chance of having cardiovascular disease in near-future and greater mortality rates among these subjects (27). HbAlc reflects the previous 8-10 weeks glycemic index in an individual and poor glycemic control is known to be associated with elevated cholesterol, triglycerides, and low-density lipoprotein. Therefore, estimating $\mathrm{HbAlc}$ at an early stage can help decrease the risk of mortality and morbidity of both mother and the conceptus by early detection of maternal dyslipidemia 
and its prompt treatment. Hyperlipidemia typically occurs during normal pregnancy and alterations in lipid profile is known to occur in GDM (11). Significantly elevated levels of $\mathrm{HbAlc}$ in GDM and its relation to lipid profile were reported by several researchers $(19,22,23)$. McGrowder at al indicated $\mathrm{HbAlc}$ concentration was higher in women with GDM $(P=0.001)$; in addition, they found that TC and triglycerides were significantly high in these women ( $P=0.039$ for TC and $P=0.033$ for TG). However, HDLcholesterol was non-significantly low. Baxi et al (28) used glycated hemoglobin as a screening tool for macrosomia in GDM and suggested that an increased HbAlc value signals potentially enhanced average blood glucose level and demands immediate intensive management of GDM. The authors implemented a comparative study of all the lipid profile parameters between the healthy pregnant women and women having GDM. The results indicated that TC and low-density lipoprotein levels did not alter significantly in GDM cases; values of $185.12 \pm 22.78 \mathrm{mg} /$ $\mathrm{dL}$ and $197.40 \pm 40.53 \mathrm{mg} / \mathrm{dL}$ were obtained for GDM and normal pregnancies, respectively, along with a $P$-value more than 0.05 for TC; Further, values of $111.73 \pm 17.26$ $\mathrm{mg} / \mathrm{dL}$ and $114.22 \pm 35.64 \mathrm{mg} / \mathrm{dL}$ were estimated for GDM and normal pregnancies, as well as a $P$ value more than 0.05 for LDL-cholesterol. These results are in agreement with the findings of Hollingsworth and Grundy (29), and Arcos et al (30), where they found an insignificant correlation between $\mathrm{HbA1c}$ with TC and LDL-cholesterol in GDM and normal pregnancies. Hollingsworth and Grundy indicated that mean plasma levels of TC and LDL-cholesterol in insulin-dependent diabetic patients (type I) did not differ from those of healthy pregnancies. Furthermore, Emet et al (31) studied plasma lipids and lipoproteins during pregnancy and found the change in TC and LDL-cholesterol was negligible in patients with glucose intolerance. Conversely, Montelongo et al (32) reported a significant rise in TC and LDL-cholesterol in GDM groups and normal pregnant subjects. Moreover, Khan et al compared the biochemical profile of GDM subjects with non-GDM and reported a significant increase in TC $(206 \pm 18.79 \mathrm{mg} / \mathrm{dL})$ in GDM cases compared to the healthy pregnant women $(195 \pm 24.15 \mathrm{mg} / \mathrm{dL})$; however, the results of their study demonstrated no significant rise of LDL-cholesterol in GDM $(93 \pm 18.71 \mathrm{mg} / \mathrm{dL})$ compared to the control women $(88 \pm 16.35 \mathrm{mg} / \mathrm{dL})$.

Additionally, the resultsofthecurrentstudydemonstrated a significant decrease in HDL-cholesterol while indicating a significant increase in TG: HDL-cholesterol ratio in GDM cases compared to the healthy pregnant women with a $P<0.001$ in the case of HDL-cholesterol and $P<0.01$ in the case of TG: HDL-cholesterol ratio. Although TG levels in the present study revealed no significant increase in GDM pregnancies, it is on the higher side when compared to TG levels in normal pregnant women (122.52 \pm 51.50 $\mathrm{mg} / \mathrm{dL}$ in the case of GDM and $105.26 \pm 41.70 \mathrm{mg} / \mathrm{dL}$ in normal pregnant women). In addition, increased levels of triglyceride were reported by Kjos et al (11). Another study by Aura et al (33) evaluated the incidence of impaired lipid profile parameters in subjects with GDM and their relationship with gestation, fetal complications, and metabolic syndrome variables of the mother; they reported decreased HDL-cholesterol levels while increased TG levels in GDM cases. Further, Ryckman et al (34) observed that triglycerides significantly increased among female subjects diagnosed with GDM in contrast to the healthy ones. Furthermore, HDL-C levels significantly decreased in GDM cases compared to those without GDM in the second and third trimesters of pregnancy. However, Sobki at al (35) concluded that lipid parameters in the serum of pregnant subjects demonstrated no significant difference between GDM patients and healthy females. By estimating the serum TG, HDL-cholesterol, and most importantly, TG: HDL-cholesterol ratio, the severity of abnormal maternal metabolism, as well as the degree of dyslipidemia can be predicted which, therefore, leads to better antenatal care for avoiding further complications both for the mother and the neonate. Moreover, in one of the findings of American Heart Association, it was revealed that the chance of having heart diseases was significantly increased in people having high ratios of triglyceride and HDL cholesterol compared to those having lower ratios (36).

The limitations of the study included the small sample size. A larger number of participants in the study provides more comprehensive results. Additionally, the present study was not extended throughout the pregnancy, namely, until the end of the third trimester. This posed a restriction for generalizing the association between $\mathrm{HbAlc}$ and lipid profile among pregnant women at all gestational ages. It would be interesting to follow the GDM pregnancies through each trimester to better correlate the blood glucose levels with maternal lipid metabolism.

\section{Conclusions}

The results of the study prove beyond doubt that blood levels of glucose, glycated hemoglobin, triglycerides, and high-density lipoprotein play significantly important roles in developing dyslipidemia in GDM state. Although it is known that lipid parameters increase even in normal pregnancy, how it increases in GDM is different; In addition, based on the results, a significant decrease does not typically occur in HDL-cholesterol in normal pregnancies. We need a highly larger sample size in order to further validate the results and demonstrate that glycated hemoglobin can be exploited as a potential index of abnormal lipid profile in GDM.

\section{Conflict of Interests}

Authors declare that they have no conflict of interests.

\section{Financial Support}

No specific funding was received for this study. 


\section{References}

1. Metzger BE, Coustan DR. Summary and recommendations of the Fourth International Workshop-Conference on Gestational Diabetes Mellitus. The Organizing Committee. Diabetes Care. 1998;21 Suppl 2:B161-167.

2. Ryan EA, Enns L. Role of gestational hormones in the induction of insulin resistance. J Clin Endocrinol Metab. 1988;67(2):341-347. doi:10.1210/jcem-67-2-341

3. Sonagra AD, Biradar SM, K D, Murthy DSJ. Normal pregnancy- a state of insulin resistance. J Clin Diagn Res. 2014;8(11):Cc01-03. doi:10.7860/jcdr/2014/10068.5081

4. Sattar N, Greer IA, Louden J, et al. Lipoprotein subfraction changes in normal pregnancy: threshold effect of plasma triglyceride on appearance of small, dense low density lipoprotein. J Clin Endocrinol Metab. 1997;82(8):24832491. doi:10.1210/jcem.82.8.4126

5. Brizzi P, Tonolo G, Esposito F, et al. Lipoprotein metabolism during normal pregnancy. Am J Obstet Gynecol. 1999;181(2):430-434.

6. Loke DF, Viegas OA, Kek LP, Rauff M, Thai AC, Ratnam SS. Lipid profiles during and after normal pregnancy. Gynecol Obstet Invest. 1991;32(3):144-147. doi:10.1159/000293016

7. Toescu V, Nuttall SL, Martin U, Kendall MJ, Dunne F. Oxidative stress and normal pregnancy. Clin Endocrinol (Oxf). 2002;57(5):609-613.

8. Little RR. Recent progress in glycohemoglobin (HbA1c) testing. Diabetes Care. 2000;23(3):265-266.

9. Selvin E, Coresh J, Golden SH, Brancati FL, Folsom AR, Steffes MW. Glycemic control and coronary heart disease risk in persons with and without diabetes: the atherosclerosis risk in communities study. Arch Intern Med. 2005;165(16):1910-1916. doi:10.1001/archinte.165.16.1910

10. VinodMahato R, Gyawali P, Raut PP, et al. Association between glycaemic control and serum lipid profile in type 2 diabetic patients: Glycated haemoglobin as a dual biomarker. Biomed Res. 2011;22(3):375-380.

11. Kjos SL, Buchanan TA, Montoro M, Coulson A, Mestman JH. Serum lipids within 36 mo of delivery in women with recent gestational diabetes. Diabetes. 1991;40 Suppl 2:142146.

12. Jiang $\mathrm{S}$, Jiang $\mathrm{J}, \mathrm{Xu} \mathrm{H}$, et al. Maternal dyslipidemia during pregnancy may increase the risk of preterm birth: A meta-analysis. Taiwan J Obstet Gynecol. 2017;56(1):9-15. doi:10.1016/j.tjog.2016.07.012

13. Simmons LE, Rubens CE, Darmstadt GL, Gravett MG. Preventing preterm birth and neonatal mortality: exploring the epidemiology, causes, and interventions. Semin Perinatol. 2010;34(6):408-415. doi:10.1053/j. semperi.2010.09.005

14. Roggenbuck LF, Kleinwechter HJ, Demandt N, Dorner KM. Diagnostics of gestational diabetes: which cutoff-values are valid for capillary whole blood? Clin Lab. 2004;50(7-8):403408.

15. Gilmartin AB, Ural SH, Repke JT. Gestational diabetes mellitus. Rev Obstet Gynecol. 2008;1(3):129-134.

16. Ozder A. Lipid profile abnormalities seen in T2DM patients in primary healthcare in Turkey: a cross-sectional study. Lipids Health Dis. 2014;13:183. doi:10.1186/1476511x-13-183

17. Schaefer-Graf UM, Pawliczak J, Passow D, et al. Birth weight and parental BMI predict overweight in children from mothers with gestational diabetes. Diabetes Care. 2005;28(7):1745-1750.

18. Schaefer-Graf UM, Graf K, Kulbacka I, et al. Maternal lipids as strong determinants of fetal environment and growth in pregnancies with gestational diabetes mellitus. Diabetes Care. 2008;31(9):1858-1863. doi:10.2337/dc08-0039

19. Lenin M, Ramesh R, Kuzhandai Velu V, Ghose S. Association of dyslipidemia and Glycated hemoglobin in gestational diabetes mellitus. J Diabetes Mellitus. 2017;7(4):275-280. doi:10.4236/jdm.2017.74022

20. Bleicher SJ, O'Sullivan JB, Freinkel N. Carbohydrate metabolism in pregnancy: The interrelations of glucose, insulin and free fatty acids in late pregnancy and post partum. N Engl J Med. 1964;271:866-872. doi:10.1056/ nejm196410222711702

21. Taricco E, Radaelli T, Rossi G, et al. Effects of gestational diabetes on fetal oxygen and glucose levels in vivo. BJOG. 2009;116(13):1729-1735. doi:10.1111/j.14710528.2009.02341.x

22. Khan R, Ali K, Khan Z, Ahmad T. Lipid profile and glycosylated hemoglobin status of gestational diabetic patients and healthy pregnant women. Indian J Med Sci. 2012;66(7-8):149-154. doi:10.4103/0019-5359.114175

23. Ghafoor S, Shaikh AW, Shaheena. Maternal lipids in pregnancies with gestational diabetes mellitus. Pak J Med Health Sci. 2012;6(1):81-84.

24. McGrowder D, Grant K, Irving R, et al. Lipid profile and clinical characteristics of women with gestational diabetes mellitus and preeclampsia. J Med Biochem. 2009;28(2):7281. doi:10.2478/v10011-009-0007-x

25. Khan HA, Sobki SH, Khan SA. Association between glycaemic control and serum lipids profile in type 2 diabetic patients: HbA1c predicts dyslipidaemia. Clin Exp Med. 2007;7(1):24-29. doi:10.1007/s10238-007-0121-3

26. Martin-Timon I, Sevillano-Collantes C, Segura-Galindo A, Del Canizo-Gomez FJ. Type 2 diabetes and cardiovascular disease: Have all risk factors the same strength? World J Diabetes. 2014;5(4):444-470. doi:10.4239/wjd.v5.i4.444

27. Selvin E, Steffes MW, Zhu H, et al. Glycated hemoglobin, diabetes, and cardiovascular risk in nondiabetic adults. N Engl J Med. 2010;362(9):800-811. doi:10.1056/ NEJMoa0908359

28. Baxi L, Barad D, Reece EA, Farber R. Use of glycosylated hemoglobin as a screen for macrosomia in gestational diabetes. Obstet Gynecol. 1984;64(3):347-350.

29. Hollingsworth DR, Grundy SM. Pregnancy-associated hypertriglyceridemia in normal and diabetic women. Differences in insulin-dependent, non-insulin-dependent, and gestational diabetes. Diabetes. 1982;31(12):1092-1097.

30. de Arcos F, Castelo-Branco C, Casals E, Sanllehy C, Cararach V. Normal and gestational diabetic pregnancies. Lipids, lipoproteins and apolipoproteins. J Reprod Med. 1998;43(2):144-148.

31. Emet T, Ustuner I, Guven SG, et al. Plasma lipids and lipoproteins during pregnancy and related pregnancy outcomes. Arch Gynecol Obstet. 2013;288(1):49-55. doi:10.1007/s00404-013-2750-y

32. Montelongo A, Lasuncion MA, Pallardo LF, Herrera E. Longitudinal study of plasma lipoproteins and hormones 
during pregnancy in normal and diabetic women. Diabetes. 1992;41(12):1651-1659.

33. Herrera Martinez A, Palomares Ortega R, Bahamondes Opazo R, Moreno-Moreno P, Molina Puerta Mf, GalvezMoreno MA. Hyperlipidemia during gestational diabetes and its relation with maternal and offspring complications. Nutr Hosp. 2018;35(3):698-706. doi:10.20960/nh.1539

34. Ryckman KK, Spracklen CN, Smith CJ, Robinson JG, Saftlas AF. Maternal lipid levels during pregnancy and gestational diabetes: a systematic review and meta-analysis. BJOG.
2015;122(5):643-651. doi:10.1111/1471-0528.13261

35. Sobki SH, Al-Senaidy AM, Al-Shammari TA, Inam SS, AlGwiser AA, Bukhari SA. Impact of gestational diabetes on lipid profiling and indices of oxidative stress in maternal and cord plasma. Saudi Med J. 2004;25(7):876-880.

36. Gaziano JM, Hennekens CH, O’Donnell CJ, Breslow JL, Buring JE. Fasting triglycerides, high-density lipoprotein, and risk of myocardial infarction. Circulation. 1997;96(8):2520-2525.

(C) 2019 The Author (s); This is an open-access article distributed under the terms of the Creative Commons Attribution License (http://creativecommons.org/licenses/by/4.0), which permits unrestricted use, distribution, and reproduction in any medium, provided the original work is properly cited. 